\title{
Error Analysis in Two-Terminal Impedance Measurements With Residual Correction
}

\author{
Josep M. Torrents, Member, IEEE, and Ramon Pallàs-Areny, Fellow, IEEE
}

\begin{abstract}
Residual impedance correction in impedance analyzers when using an asymmetrical test fixture needs three reference measurements, usually open circuit, short circuit, and load (meaning an impedance close to the impedance under test). This paper provides an error estimate for impedance measurements that apply a simple open/short correction in spite of using an asymmetrical test fixture. Experimental results show that the minimal error is obtained for impedance values close to the geometric mean of the short-circuit and open-circuit impedances, and that the theoretical prediction is indeed an upper limit for the actual error.
\end{abstract}

Index Terms-Error analysis, impedance measurements, residual correction.

\section{INTRODUCTION}

A CCURATE impedance measurements require us to consider the effects of residual impedance in test fixtures. Test fixtures can be modeled as two-port networks described by their transmission parameters $(A, B, C, D)$ [1]. Usually, test fixtures are symmetrical (balanced), so that $A=D$ and they can be described by two ratios between transmission parameters. These ratios can be determined from two reference measurements, for example, open-circuit and short-circuit conditions (open/short correction), and the results allow us to correct for the unknown residual impedance in electric contacts and cables connecting the impedance under test to the impedance analyzer. When the test fixture is not symmetrical, residual correction needs three reference measurements because we have to determine three ratios between transmission parameters [2]. Usually, a reference impedance close to the impedance under test is measured, hence the name open/short/load correction.

However, it is sometimes difficult to obtain reference impedances close to the impedance under test, for example, when measuring electrolytes. Therefore, the question arises about the error associated to impedance measurements that use an asymmetrical test fixture but implement a simple open/short correction procedure. This paper provides an upper limit for the error in such impedance measurements and substantiates the predictions by experimental results obtained in two-wire impedance measurements.

Manuscript received June 11, 2004; revised February 10, 2005. This work was supported by the Spanish Ministry of Science and Technology under Project TAP99-0742.

The authors are with the Technical School of Castelldefels (EPSC), Department of Electronic Engineering, Technical University of Catalonia (UPC), 08034 Barcelona, Spain (e-mail: torrents@eel.upc.edu).

Digital Object Identifier 10.1109/TIM.2005.853671

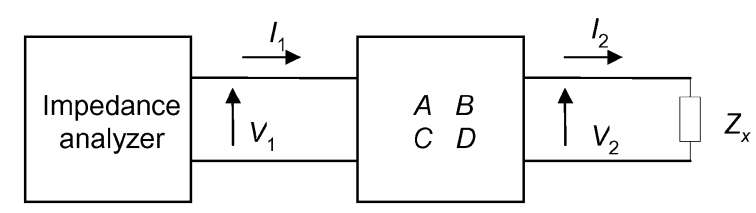

Fig. 1. Test fixture connecting the impedance under test to the impedance analyzer is modeled by a two-port network described by its four transfer parameters $A, B, C, D$

\section{ERROR MODEL IN TWO-TERMINAL IMPEDANCE MEASUREMENTS}

Fig. 1 shows the impedance under test connected to an impedance analyzer through a test fixture modeled by its transfer parameters $A, B, C$, and $D$. The voltage and current at the input of the impedance meter can be obtained from

$$
\left(\begin{array}{l}
V_{1} \\
I_{1}
\end{array}\right)=\left(\begin{array}{ll}
A & B \\
C & D
\end{array}\right)\left(\begin{array}{l}
V_{2} \\
I_{2}
\end{array}\right) .
$$

The measured impedance, disregarding the uncertainty of the impedance meter, will be

$$
Z_{x m}=\frac{V_{1}}{I_{1}}=\frac{A V_{2}+B I_{2}}{C V_{2}+D I_{2}}
$$

whereas the actual impedance under test is

$$
Z_{x}=\frac{V_{2}}{I_{2}} .
$$

In order to estimate $Z_{x}$ from $Z_{x \mathrm{~m}}$, we first rewrite (2) by replacing $V_{2} / I_{2}=Z_{\mathrm{x}}$ to obtain

$$
Z_{x m}=\frac{A Z_{x}+B}{C Z_{x}+D} .
$$

Solving for $Z_{x}$ yields

$$
Z_{x}=\frac{D Z_{x m}-B}{A-C Z_{x m}} .
$$

Measuring at open-circuit condition $\left(I_{2}=0\right)$, (2) yields $Z_{\text {om }}=$ $A / C$. Measuring at short-circuit condition $\left(V_{2}=0\right)$, (2) yields $Z_{s m}=B / D$. Therefore, (5) can be rewritten as

$$
Z_{x}=\frac{D}{A} \frac{Z_{x m}-Z_{s m}}{1-\frac{Z_{x m}}{Z_{o m}}} .
$$

If we measure a known impedance $Z_{1}$ and obtain a result $Z_{l m}$, we will have

$$
Z_{1}=\frac{D}{A} \frac{Z_{l m}-Z_{s m}}{1-\frac{Z_{l m}}{Z_{o m}}}
$$


Solving for $D / A$ and replacing it in (6) yields

$$
Z_{x \mathrm{u}}=Z_{1} \frac{1-\frac{Z_{l m}}{Z_{o m}}}{Z_{l m}-Z_{s m}} \frac{Z_{x \mathrm{~m}}-Z_{s m}}{1-\frac{Z_{x m}}{Z_{o m}}}
$$

where the subscript "u" has been added to indicate that $Z_{x}$ has been measured with an unbalanced (asymmetrical) fixture and corrected by three reference readings: $Z_{o m}, Z_{s m}$, and $Z_{l m}$. If the network connecting $Z_{x}$ to the impedance analyzer were balanced (symmetrical), we would have $A=D$, and (6) would reduce to

$$
Z_{x \mathrm{~b}}=\frac{Z_{x m}-Z_{s m}}{1-\frac{Z_{x m}}{Z_{o m}}}
$$

where the "b" in the subscript stands for balanced. Equation (9) differs from (8) by a gain factor. Therefore, using only two reference measurements when the network connecting the impedance under test to the impedance meter is asymmetrical implies to consider a unity gain factor in (8), which is equivalent to use (9) to calculate the corrected impedance value from the reading $Z_{x \mathrm{~m}}$ and the two reference measurements $Z_{o m}$ and $Z_{s m}$, instead of using (8). Hence, the modulus of the relative error is

$$
\left|\frac{e(Z)}{Z}\right|=\left|\frac{Z_{x \mathrm{~b}}-Z_{x \mathrm{u}}}{Z_{x \mathrm{u}}}\right|=\left|\frac{1}{Z_{1}} \frac{Z_{l m}-Z_{s m}}{1-\frac{Z_{l m}}{Z_{o m}}-1}\right| .
$$

Because it is recommended to select $Z_{1}$ close to $Z_{x}$, and we can assume $Z_{x \mathrm{~m}}$ to be close to $Z_{x}$, we can approximate $Z_{1} \approx Z_{x} \approx$ $Z_{x \mathrm{~m}}$ and $Z_{l m} \approx Z_{x \mathrm{~m}}$. Under these assumptions, (10) leads to

$$
\left|\frac{e(Z)}{Z}\right| \cong\left|\frac{Z_{x m}^{2}-Z_{o m} Z_{s m}}{Z_{x m}\left(Z_{o m}-Z_{x m}\right)}\right| \text {. }
$$

In summary, disregarding measurement uncertainties in the impedance analyzer, if we use open/short impedance measurements to correct residual impedances for an unbalanced test fixture, we can estimate the true impedance value corresponding to an instrument reading $Z_{x m}$ by applying (9), but the calculated result has a maximal relative error given by (11). Furthermore, because impedance values are complex numbers, the error due to an asymmetrical test fixture will affect both the amplitude and phase of the result.

From (11), when the result is close to the geometrical mean of the open circuit and short circuit measurements

$$
\left.Z_{x m}\right|_{\mathrm{opt}}=\sqrt{Z_{o m} Z_{s m}}
$$

the error because of the unbalanced connecting network is zero. Nevertheless, this calculated impedance value is not necessarily close to any impedance of interest.

\section{EXPERIMENTAL RESULTS AND DISCUSSION}

We have applied the analysis above to predict the contribution from incompletely corrected residual impedances to the error of impedance measurements performed with the HP4294A impedance analyzer when using an unbalanced (asymmetrical) test fixture. We assessed the influence of the asymmetrical test fixture on the result by measuring several reference impedances,

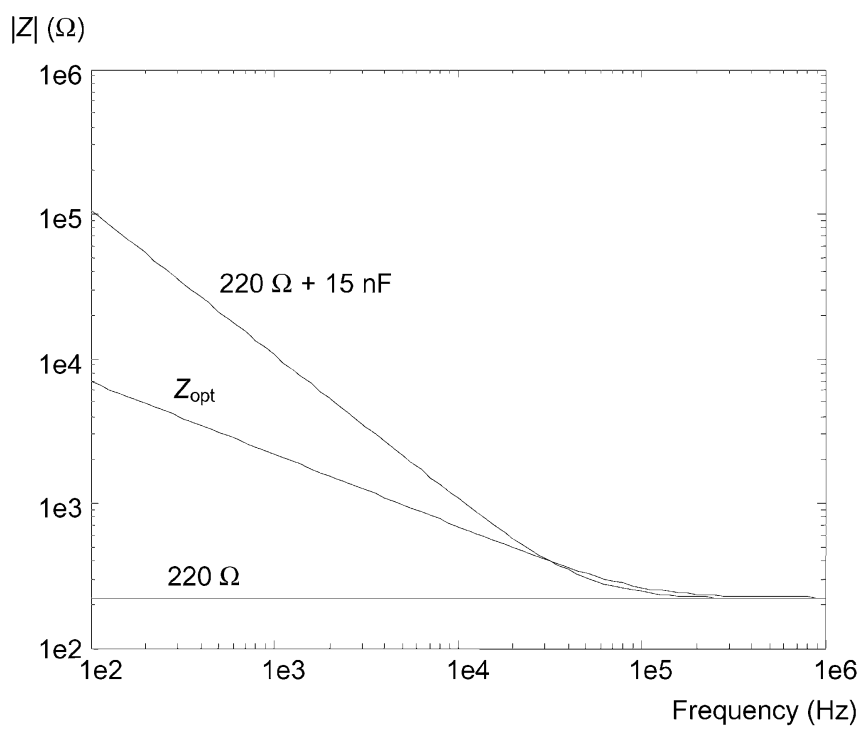

Fig. 2. Frequency dependence of the modulus of optimal impedance $Z_{\mathrm{opt}}=$ $\sqrt{ }\left(Z_{\text {om }} Z_{\text {sm }}\right)$ and of two actual impedances $220 \Omega$ and $220 \Omega$ in series with $15 \mathrm{nF}$ not far from $Z_{\mathrm{opt}}$.

first with a symmetrical test fixture provided by the manufacturer (HP16047A) and then by connecting each impedance under test to the instrument with RG214 cables 1-m long. Both measurement sets used the corresponding open/short correction. The impedance values calculated from (9) when using a symmetrical test fixture and its open/short correction were considered the "true" value $Z_{x u}$. That is, instead of applying (8) to the results obtained when using the asymmetrical test fixture and open/short/load correction, we obtained $Z_{x u}$ by using the symmetrical test fixture and open/short correction, according to (9). This alternative procedure was possible because the test impedances selected could be connected to the symmetrical test fixture.

The impedance analyzer averaged eight readings for each impedance value before transferring the results to a computer for calculation and display. Measurements were performed in the "precise mode," which implies a narrow bandwidth, and hence a long measurement time (about 5 min each ten readings). Because of this, averaging a higher number of readings could result in thermal drifts due to temperature changes in the laboratory. The impedance under test was inside a grounded Faraday cage measuring $10 \times 10 \times 12.5 \mathrm{~cm}$. Cable shields were grounded too. Because the impedance analyzer is a self-balanced bridge, the capacitance contributed by the grounded shield did not affect the measurement result [3].

Test impedance values were chosen according to the open and short-circuit measurements when using the asymmetrical test fixture. We obtained $Z_{o m}=1 /(j \omega \times 2 \mathrm{pF})$ and $Z_{s m}=$ $60 \mathrm{~m} \Omega+j \omega \times 100 \mathrm{nH}$. From these values, (12) yields an "optimal" impedance $\left(Z_{\mathrm{opt}}\right)$ that is impossible to implement in a broad frequency range. $Z_{o m}$ being capacitive, an inductive $Z_{s m}$ would result in a resistive $Z_{\text {opt }}$. However, because of the resistive component of $Z_{s m}$, contributed by the length of the cable in the asymmetrical test fixture, $Z_{\text {opt }}$ displays a $-10 \mathrm{~dB} / \mathrm{dec}$ slope (Fig. 2) at low frequency that cannot be obtained by common 
TABLE I

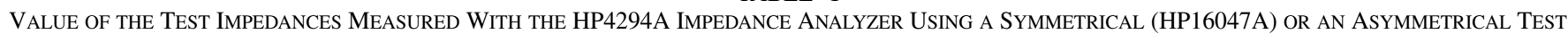
FiXture (RG214 CABLES 1-m LONG), WhEN USING AN OPEN/SHORT RESIDUAL COMPENSATION IN Both CASES

\begin{tabular}{c|c|c|c|c|c|c}
\hline \multirow{2}{*}{$\begin{array}{c}\text { Test } \\
\text { impedance }\end{array}$} & \multicolumn{5}{|c}{ Symmetrical test fixture } & \multicolumn{2}{c}{ Asymmetrical test fixture } \\
\cline { 2 - 7 } & $100 \mathrm{~Hz}$ & $10 \mathrm{kHz}$ & $1 \mathrm{MHz}$ & $100 \mathrm{~Hz}$ & $10 \mathrm{kHz}$ & $1 \mathrm{MHz}$ \\
\hline \multirow{2}{*}{$220 \Omega$} & $216-j 0.072$ & $216-j 0.0001$ & $216-j 0.095$ & $216-j 0.066$ & $216+j 0.002$ & $216-j 0.296$ \\
\hline $220 \Omega+15 \mathrm{nF}$ & $540-j 121 \times 10^{3}$ & $226-j 1.23 \times 10^{3}$ & $216-j 12.8$ & $547-j 121 \times 10^{3}$ & $226-j 1.23 \times 10^{3}$ & $216-j 13.0$ \\
\hline $10 \Omega$ & $10.00-j 0.005$ & $10.00-j 0.002$ & $10.00+j 0.049$ & $10.04-j 0.009$ & $10.05-j 0.005$ & $10.10+j 0.596$ \\
\hline $51 \Omega$ & $49.46-j 0.017$ & $49.46-j 0.008$ & $49.47+j 0.024$ & $49.50-j 0.022$ & $49.50-j 0.0002$ & $49.55+j 0.523$ \\
\hline $1 \mathrm{k} \Omega$ & $974-j 0.358$ & $974-j 0.100$ & $974-j 1.45$ & $974-j 0.337$ & $974-j 0.169$ & $973-j 13.5$ \\
\hline $5.1 \mathrm{k} \Omega$ & $5.10 \times 10^{3}-j 2.19$ & $5.10 \times 10^{3}-j 1.12$ & $5.10 \times 10^{3}-j 39.1$ & $5.10 \times 10^{3}-j 2.19$ & $5.10 \times 10^{3}-j 4.74$ & $5.07 \times 10^{3}-j 375$ \\
\hline
\end{tabular}

passive components. At higher frequencies, the inductive component of $Z_{s m}$ predominates over its resistive component, and $Z_{\mathrm{opt}} \approx(100 \mathrm{nH} / 2 \mathrm{pF})^{1 / 2}=100 \sqrt{ } 5 \Omega \approx 223 \Omega$. We have selected two impedances relatively close to that optimum: a $220-\Omega$ off-the-shelf resistor (nominal value) and the series combination of that resistor and a $15-\mathrm{nF}$ capacitor (nominal value). Fig. 2 shows the modulus of $Z_{\text {opt }}, 220 \Omega$, and $220 \Omega$ in series with $15 \mathrm{nF}$, from $100 \mathrm{~Hz}$ to $1 \mathrm{MHz}$. The remaining test impedances were also off-the-shelf resistors whose nominal values were $1 \mathrm{k} \Omega, 51 \Omega, 5.1 \mathrm{k} \Omega$, and $10 \Omega$. These values were selected far from the optimal impedance but such that the respective geometric mean of each pair $(1 \mathrm{k} \Omega, 51 \Omega),(5.1 \mathrm{k} \Omega, 10 \Omega)$ were close to $Z_{\mathrm{opt}}$.

We measured these six impedances (five resistors and one resistor in series with a capacitor) from $100 \mathrm{~Hz}$ to $1 \mathrm{MHz}$ using both the symmetrical and the asymmetrical test fixture and the open/short correction in both cases. Table I shows the results at $100 \mathrm{~Hz}, 10 \mathrm{kHz}$, and $1 \mathrm{MHz}$. The use of the open/short correction prevents, for example, that small-value resistors display an inductive component that would be contributed by the short-circuit impedance. Nevertheless, Table I shows that actual resistors have a reactive component. In general, the results obtained with the asymmetrical test fixture different more from the nominal values than those obtained with the symmetrical test fixture, as expected.

Fig. 3 shows the maximal modulus of the relative error predicted by (11) for the six test impedances in the range from $100 \mathrm{~Hz}$ to $1 \mathrm{MHz}$. The theoretical maximal error is minimum for the two impedances whose value is close to the optimum calculated from (12), namely $220 \Omega$ and $220 \Omega+15 \mathrm{nF}$, and increases with frequency for impedances far from that optimum. At high frequency, the relative error is similar for $51 \Omega$ and $1 \mathrm{k} \Omega$, and for $5.1 \mathrm{k} \Omega$ and $10 \Omega$, whose values are (geometrically) symmetrical about that given by (12). At low frequency, however, the relative error for each resistor from each pair is quite different: it is larger for $10 \Omega$ than $51 \mathrm{k} \Omega$, and larger for $51 \Omega$ than for $1 \mathrm{k} \Omega$. Furthermore, the relative error increases for impedance values

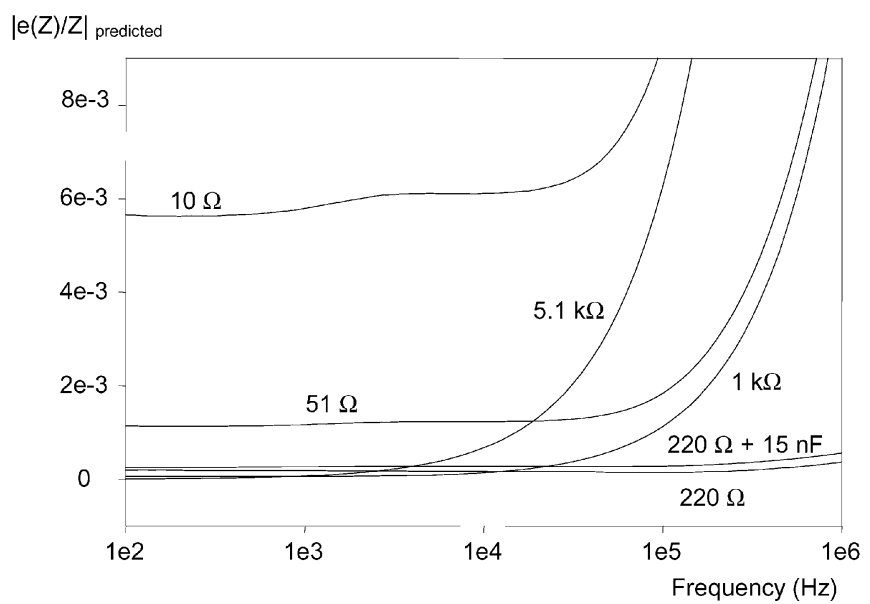

Fig. 3. Maximal modulus of the predicted relative error for the six test impedance values according to (11), from $100 \mathrm{~Hz}$ to $1 \mathrm{MHz}$.

that are very different from (12): it is larger for $5.1 \mathrm{k} \Omega$ and $10 \Omega$ than for $1 \mathrm{k} \Omega$ and $51 \Omega$.

In order to understand why, at low frequency, resistors larger than $Z_{\mathrm{opt}}$ have a reduced relative error compared to resistors smaller than $Z_{\mathrm{opt}}$, Fig. 4 plots (11) for different measured resistors $\left(Z_{x m}\right)$, at five frequencies. These plots confirm the result in Fig. 3 and can be explained as follows: the denominator in (11) increases whenever $Z_{x m}$ or $Z_{\mathrm{om}}-Z_{x m}$ are small, which at high frequencies means a very low or a very high measured impedance, compared to $Z_{\mathrm{opt}}$. At low frequency, however, $Z_{o m}-Z_{x m}$ is large even for a high $Z_{x m}$ because $Z_{o m}$ is reactive and $Z_{x m}$ is resistive (that is, at low frequency, the reactive component of the resistor is small). Hence, for the instrument and test fixtures used, at low frequency, low resistors yield a large relative error but high resistors yield a small relative error.

Fig. 5 shows the modulus of the relative error of the impedance for the six test impedances measured in the range from $100 \mathrm{~Hz}$ to $1 \mathrm{MHz}$. The relative error is minimal for $220 \Omega$ 
$|\mathrm{e}(\mathrm{Z}) / \mathrm{Z}|$

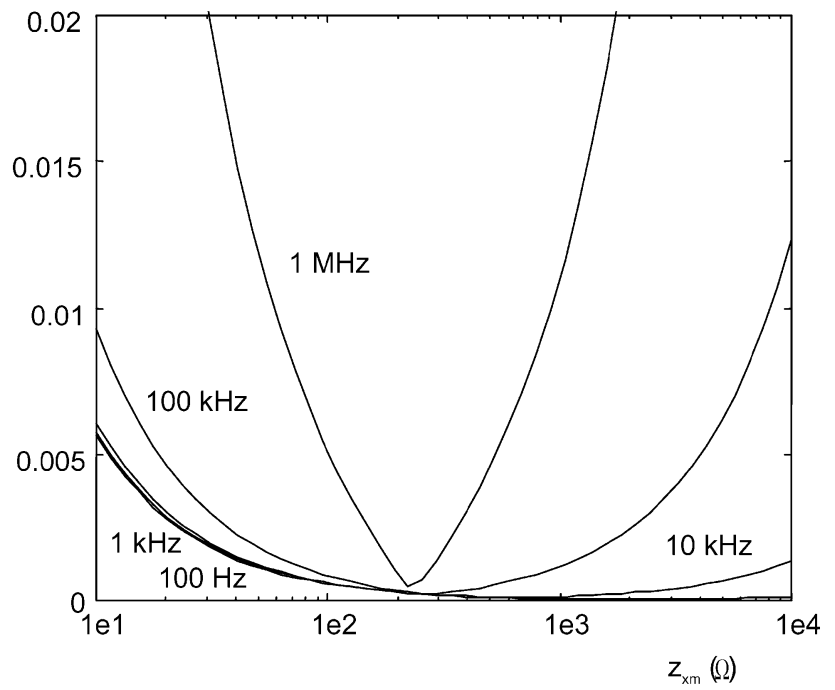

Fig. 4. Maximal modulus of the predicted relative error according to (11) for different measured impedances $Z_{x m}$ at five different frequencies.

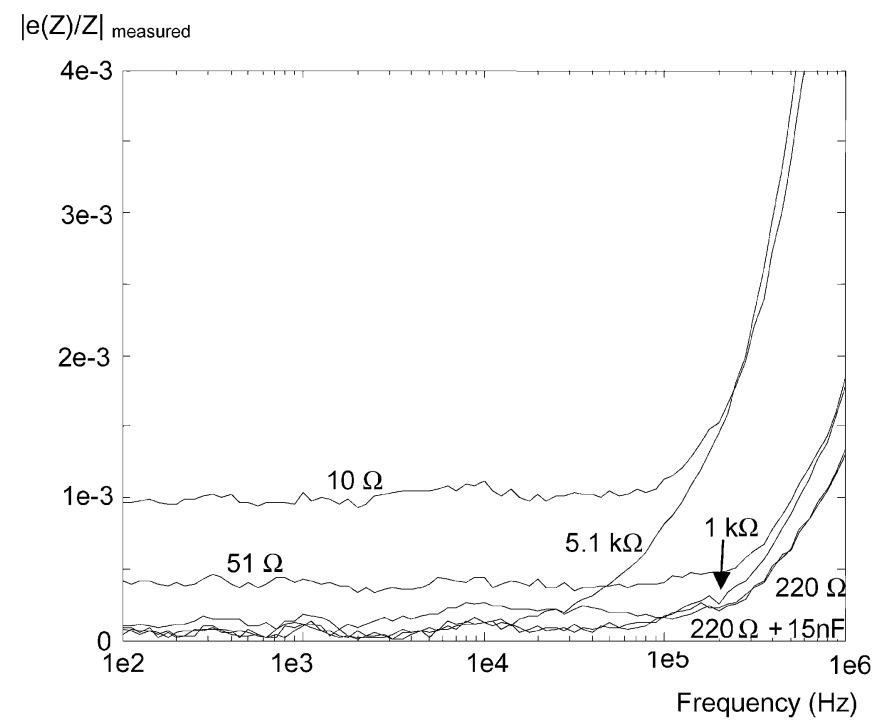

Fig. 5. Modulus of the relative error for the six test impedance values measured with the HP4294A.

and $220 \Omega+15 \mathrm{nF}$, as predicted, and is smaller than the predicted error in Fig. 3. The relative error increases from $100 \mathrm{kHz}$ and up, particularly for the highest and lowest impedance values $(5.1 \mathrm{k} \Omega$ and $10 \Omega$ ), whereas the predicted relative error increases above $10 \mathrm{kHz}$. At high frequency, the error is similar for $51 \Omega$ and $1 \mathrm{k} \Omega$, the same as in Fig. 3. Also, the relative error is smaller for $5.1 \mathrm{k} \Omega$ than for $10 \Omega$, particularly at low frequency, which is consistent with the theoretical prediction.

\section{CONCLUSION}

Accurate measurements using impedance analyzers require us to compensate for impedance residuals in the test fixture. When the test fixture is symmetrical, this correction is usually performed by measuring short-circuit and open-circuit impedance and using the results to compute the actual impedance according to (9). When the text fixture is asymmetrical, we need a third reference measurement in order to compensate for impedance residuals according to (8). Using simple open/short correction instead adds gain error to that of the impedance analyzer. Equation (11) estimates the maximal relative error added, and (12) determines the impedance value having the minimal relative error. Experimental results confirm that 1) actual relative errors when performing simple open/short correction are smaller than the theoretical limit calculated from $(11), 2$ ) relative errors for impedance values close to the optimum calculated from (12) are very small, and 3) relative errors for the HP4294A impedance analyzer and a test fixture consisting of RG214 cables 1-m long, are larger for low impedance values than for high impedance values.

\section{REFERENCES}

[1] M. Honda, The Impedance Measurement Handbook. A Guide to Measurement Technology and Techniques. Tokyo, Japan: Yokogawa-Hewlett-Packard Ltd., 1989.

[2] Effective Impedance Measurement Using Open/Short/Load Correction, 1998. Application Note 346-3. Hewlett Packard, Palo Alto, CA.

[3] Y. Narimatsu, Electronic Instrument Handbook, 2nd ed, C. F. Coombs, Ed. New York: McGraw-Hill, 1995, ch. 7. Impedance measuring instruments.

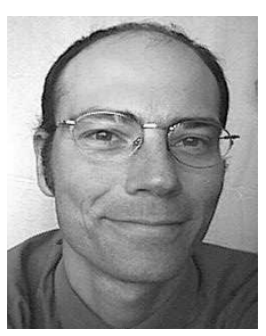

Josep M. Torrents (M'96) received the Enginyer de Telecomunicació and Doctor Enginyer de Telecomunicació degrees from the Technical University of Catalonia (UPC), Barcelona, Spain, in 1989 and 1996, respectively.

$\mathrm{He}$ is an Associate Professor of electronic engineering at the Technical University of Catalonia, and he teaches courses in several areas of electronics. From 1998 to 1999 , he was a Visiting Scholar at Northwestern University, Evanston, IL. His research includes instrumentation methods and sensors based on electrical impedance measurements, mainly applied in soil, ceramic materials, and oils. He is also interested in applications related with sustainability.

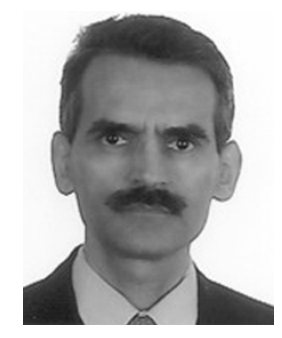

Ramon Pallàs-Areny (M'81-SM'88-F'98) received the Ingeniero Industrial and Doctor Ingeniero Industrial degrees from the Technical University of Catalonia (UPC), Barcelona, Spain, in 1975 and 1982, respectively.

$\mathrm{He}$ is a Professor of electronic engineering at the Technical University of Catalonia and teaches courses in medical and electronic instrumentation. In 1989 and 1990, he was a visiting Fulbright Scholar and, in 1997 and 1998, he was an Honorary Fellow at the University of Wisconsin, Madison. In 2001, he was nominated Professor Honoris Causa by the Faculty of Electrical Engineering, University of Cluj-Napoca, Cluj, Romania. His research includes instrumentation methods and sensors based on electrical impedance measurements, sensor interfaces, noninvasive physiological measurements, and electromagnetic compatibility in electronic systems. He is the author of several books on instrumentation in Spanish and Catalan, the latest one being Sensors and Interfaces, Solved Problems (Barcelona, Spain: Edicions UPC, 1999). $\mathrm{He}$ is also coauthor (with J. G. Webster) of Sensors and Signal Conditioning, 2nd ed. (New York: Wiley, 2001), and Analog Signal Processing (New York: Wiley, 1999).

Dr. Pallás-Areny was a recipient, with J. G. Webster, of the 1991 Andrew R. Chi Prize Paper Award from the IEEE Instrumentation and Measurement Society. In 2000, he received the Award for Quality in Teaching granted by the Board of Trustees of the UPC and, in 2002, the Narcís Monturiol Medal from the Autonomous Government of Catalonia. 